ARTICLE

https://doi.org/10.1038/s41467-019-09093-1

\title{
Impacts of alkaline on the defects property and crystallization kinetics in perovskite solar cells
}

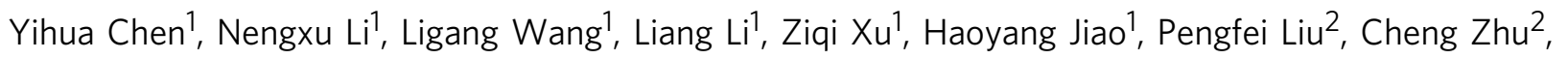
Huachao Zai ${ }^{2}$, Mingzi Sun ${ }^{3}$, Wei Zou ${ }^{4}$, Shuai Zhang ${ }^{5}$, Guichuan Xing ${ }^{6}$, Xinfeng Liu (1) ${ }^{5}$, Jianpu Wang (1) ${ }^{4}$, Dongdong $\mathrm{Li}^{7}$, Bolong Huang ${ }^{3}$, Qi Chen (i) ${ }^{2}$ \& Huanping Zhou ${ }^{1}$

Further minimizing the defect state density in the semiconducting absorber is vital to boost the power conversion efficiency of solar cells approaching Shockley-Queisser limit. However, it lacks a general strategy to control the precursor chemistry for defects density reduction in the family of iodine based perovskite. Here the alkaline environment in precursor solution is carefully investigated as an effective parameter to suppress the incident iodine and affects the crystallization kinetics during film fabrication, via rationale adjustment of the alkalinity of additives. Especially, a 'residual free' weak alkaline is proposed not only to shrink the bandgap of the absorber by modulating the stoichiometry of organic cation, but also to improve the open circuit voltage in the resultant device. Consequently, the certified efficiency of $20.87 \%$ (Newport) is achieved with one of the smallest voltage deficits of $413 \mathrm{mV}$ in the planar heterojunction perovskite solar cell.

\footnotetext{
${ }^{1}$ Key Laboratory for the Physics and Chemistry of Nanodevices, Beijing Key Laboratory for Theory and Technology of Advanced Battery Materials, Department of Materials Science and Engineering, College of Engineering, Peking University, Beijing 100871, P. R. China. ${ }^{2}$ School of Material Science and Engineering, Beijing Institute of Technology, Beijing 100081, P. R. China. ${ }^{3}$ Department of Applied Biology and Chemical Technology, Hong Kong Polytechnic University, Hong Kong 999077, P. R. China. ${ }^{4}$ Key Laboratory of Flexible Electronics \& Institute of Advanced Materials, Jiangsu National Synergetic Innovation Center for Advanced Materials, Nanjing Tech University, Nanjing 211816, P. R. China. ${ }^{5}$ CAS Key Laboratory of Standardization and Measurement for Nanotechnology, CAS Center for Excellence in Nanoscience, National Center for Nanoscience and Technology, Beijing 100190, P. R. China. ${ }^{6}$ Joint Key Laboratory of the Ministry of Education, Institute of Applied Physics and Materials Engineering, University of Macau, Macau 999078, P. R. China. ${ }^{7}$ Shanghai Advanced Research Institute, Chinese Academy of Sciences, Shanghai 201210, P. R. China. Correspondence and requests for materials should be addressed to H.Z. (email: happy_zhou@pku.edu.cn)
} 
O rganic-inorganic hybrid perovskite materials have attracted broad attention ${ }^{1}$, owing to their low fabrication cost and intriguing optical and electronic properties ${ }^{2-5}$. Impressively, it witnessed the power conversion efficiency (PCE) of perovskite solar cells boosted from $3.8 \%{ }^{6}$ to a certified $23.7 \%$ rapidly $^{7-10}$. To enable a truly competitive PCE of perovskite solar cells as that of the most efficient inorganic counterpart, e.g., $\mathrm{GaAs}$, it is required to further reduce their energy loss. This relies on the improved charge generation and mitigated non-radiative charge recombination within the complete device. With careful investigations on the absorber materials ${ }^{11,12}$, device configuration $^{13,14}$, and relevant interfaces ${ }^{15,16}$, it is found that the intrinsic defect properties ${ }^{17,18}$ in perovskite films influence the charge dynamics in the devices significantly. Halide perovskites are generally regarded as relatively soft ionic solids, which are prone to contain point defects ${ }^{3,19}$ in polycrystalline films (e.g., vacancies, interstitials, and cation and anti-site substitutions). They often serve as the non-radiative recombination centers to affect the quantum efficiency of photoluminescence and deteriorate the photovoltaic performance ${ }^{17,20}$. Therefore, to assure the best power output by reducing energy and voltage loss, it is of great importance to gain profound understanding on the defect physics and chemistry, which results in defects elimination in perovskite films for efficient photo-carrier transport.

During the past few years, efforts have been attempted to probe the defects to unveil their nature. In the methylammonium lead iodide $\left(\mathrm{MAPbI}_{3}\right)$ perovskite, it reported a variety of shallow native point defects, e.g., $\mathrm{Pb}$ vacancies $\left(\mathrm{V}_{\mathrm{Pb}}\right)$, MA vacancies $\left(\mathrm{V}_{\mathrm{MA}}\right)$, interstitials $\mathrm{MA}\left(\mathrm{MA}_{\mathrm{i}}\right)$, etc ${ }^{3}$, and deep energy-level defects $^{2}$ in forms of complex and derivatives, such as interstitials I $\left(\mathrm{I}_{\mathrm{i}}\right)$ and its derivatives ${ }^{21,22}$. In mixed perovskites, it reveals even more complicated defect physics, wherein formamidinium (FA)related defect $\mathrm{I}_{\mathrm{FA}}$ (the complexes of $\mathrm{I}_{\mathrm{i}}$ and $\mathrm{V}_{\mathrm{FA}}$ ) makes a transition at a Fermi level that sits deep within the bandgap ${ }^{19}$. Furthermore, defect formation is reported to be correlated to the processing conditions, wherein films grown under iodine-rich conditions exhibit a higher defect density ${ }^{23}$. Among all defects, iodine-related defects receive most interest. It is reported that polyiodide complexes in dimethylformamide (DMF) solution could easily induce defects due to a nonstoichiometric $\mathrm{Pb} / \mathrm{I}$ ratio in the resultant films ${ }^{24}$. When dimethyl sulfoxide (DMSO) was further introduced, either I-rich or I-deficient intermediates were identified, depending on the ratio of the starting materials ${ }^{25}$. Recently, it demonstrated iodine redox chemistry to convert hole traps into electron traps possibly by oxidation during film processing ${ }^{26}$. Subsequently, the iodide management was proposed to effectively decrease the concentration of mid-gap defects in the perovskite films, and improve the PCEs of perovskite solar cells $^{27-29}$. Yet, it lacks a feasible approach to be universally adopted in various halide perovskites, which suppresses the incidental $I_{2}$ in the absorber for enhanced device performance with reduced energy and voltage loss.

In this work, we demonstrate a universal technique to suppress the incident $I_{2}$ in the halide precursor solution by employing alkaline, wherein iodine can be effectively eliminated via a disproportionation reaction under a number of different alkaline environments. The alkalinity is further demonstrated to affect the crystallization kinetics and optoelectronic properties of perovskite films. For instance, formamidine acetate as a 'residual free' weak alkaline, is found to effectively manipulate the stoichiometry of the cations in the mixed perovskite ( $\left.(\mathrm{FA}, \mathrm{MA}, \mathrm{Cs}) \mathrm{Pb}(\mathrm{I}, \mathrm{Br})_{3}\right)$ precursor, and in particular to reduce the density of deep-level defects in the resultant films substantially. Consequently, the corresponding mixed perovskite solar cells achieve an average PCE of $20.87 \%$ (certified). More interestingly, this modified absorber possesses a smaller bandgap, but the resultant device revealed unambiguous improvement in the open-circuit voltage ( $\left.V_{\mathrm{OC}}\right)$, corresponding to a $V_{\mathrm{OC}}$ deficit of $413 \mathrm{mV}$, which is one of the smallest certified value in the planar perovskite solar cells. These findings provide a universal approach for defect reduction in iodide-based perovskite films, which substantially reduce the energy and voltage loss to boost the efficiency of the devices.

\section{Results}

Suppression of $I_{2}$ impurity via alkaline additives. Precise control of precursor solutions is crucial for perovskite film deposition. Generally, the organic precursors are hygroscopic and unstable in air or under illumination. In particular, formamidine iodide (FAI) and methylammonium iodide (MAI) can be easily oxidized to form $\mathrm{I}_{2}$ and/or other by-products, which induces the nonstoichiometric ratio in the precursor solution. It further leads to defect states in perovskite films that eventually deteriorate device performance. The degradation mechanism involves the formation of $\mathrm{I}_{2}$, which is thoroughly studied in previous work ${ }^{30}$. Ultraviolet-visible (UV-vis) absorption spectroscopy was conducted to semiquantitatively investigate the formation of iodide species in the precursor solution ${ }^{27-29,31}$. As shown in Fig. 1a, the organic cation solution used to prepare the perovskite films shows a characteristic peak at $360 \mathrm{~nm}$. It is often attributed to the absorption of $\mathrm{I}_{3}{ }^{-}$, stemmed from $\mathrm{I}^{-}$reacting reversibly with $\mathrm{I}_{2}$. Interestingly, the peak intensity equals to that of $10^{-4} \mathrm{M}$ $\mathrm{I}_{2}$ in isopropanol, indicating the trace amount of $\mathrm{I}_{2}$ in the organic cation precursor solution.

To eliminate the incident $I_{2}$ in the precursor solution, we propose to create an alkaline environment by introducing additives in the precursor solution. A series of alkaline additives with different alkalinity were employed, such as formamidine acetate (FAAc, $\left.\mathrm{p} K_{a} \sim 7.64\right)$, sodium acetate $\left(\mathrm{CH}_{3} \mathrm{COONa}, \mathrm{p} K_{a}\right.$ 9.24), sodium bicarbonate $\left(\mathrm{NaHCO}_{3}, \mathrm{p} K_{a} \sim 10.33\right)$, sodium hydroxide $\left(\mathrm{NaOH}, \mathrm{p} K_{a} \sim 14.56\right)$, and potassium hydroxide $(\mathrm{KOH}$, $\left.\mathrm{p} K_{a} \sim 14.7\right)$, to create the full spectrum of alkalinity precursor environment in the context of the alkalinity (see Supplementary Notes 1 and 2 for details). Take the alkaline solution of FAAcl ethanol for example, we observed that along with an increasing amount of FAAc, the absorbance at $360 \mathrm{~nm}$ for precursor solution was dramatically reduced (Fig. 1a). Similarly, reduced absorbance at $360 \mathrm{~nm}$ was also observed when FAAc was added to the iodine solution $\left(10^{-4} \mathrm{M}\right.$, Supplementary Fig. 1). This indicates that the diminishing absorbance at $360 \mathrm{~nm}$ in the precursor solution is due to the reduction of incident $\mathrm{I}_{2}$. Furthermore, $\mathrm{CH}_{3} \mathrm{COONa}, \mathrm{NaHCO}_{3}, \mathrm{NaOH}$, and $\mathrm{KOH}$ took the same effects as expected when added to the precursor solution (Fig. 1b-e). Meanwhile, the addition of pure ethanol does not reduce the absorption peak of $\mathrm{I}_{3}-$ (Supplementary Fig. 2). The disappearance of $\mathrm{I}_{3}{ }^{-}$(mostly related to $\mathrm{I}_{2}$ ) in the alkaline environment possibly suggests a disproportionation reaction in the precursor solution, wherein $\mathrm{I}_{2}$ is converted into iodate and iodide $^{32}$ (see Supplementary Note 3). In addition (Fig. 1f), we found that the alkalinity of additives would largely influence the disproportionation reaction. It requires less amount of $\mathrm{NaOH}$ or $\mathrm{KOH}$ to eliminate the $\mathrm{I}_{2}$ peak of precursor solution than that of FAAc, $\mathrm{CH}_{3} \mathrm{COONa}$, or $\mathrm{NaHCO}_{3}$, mostly because the former ones are 'strong' alkaline in the context of conventional inorganic chemistry.

Morphology, phase, and carrier lifetime analysis. The typical two-step approach was employed to fabricate the perovskite films (see details in the Methods section). We named the two-step processed reference perovskite film ((FA,MA,Cs) $\left.\mathrm{Pb}(\mathrm{I}, \mathrm{Br})_{3}\right)$ as the PVSK film. To ascertain the impact of alkaline additives on the morphology and crystallographic structure of the resultant films, 

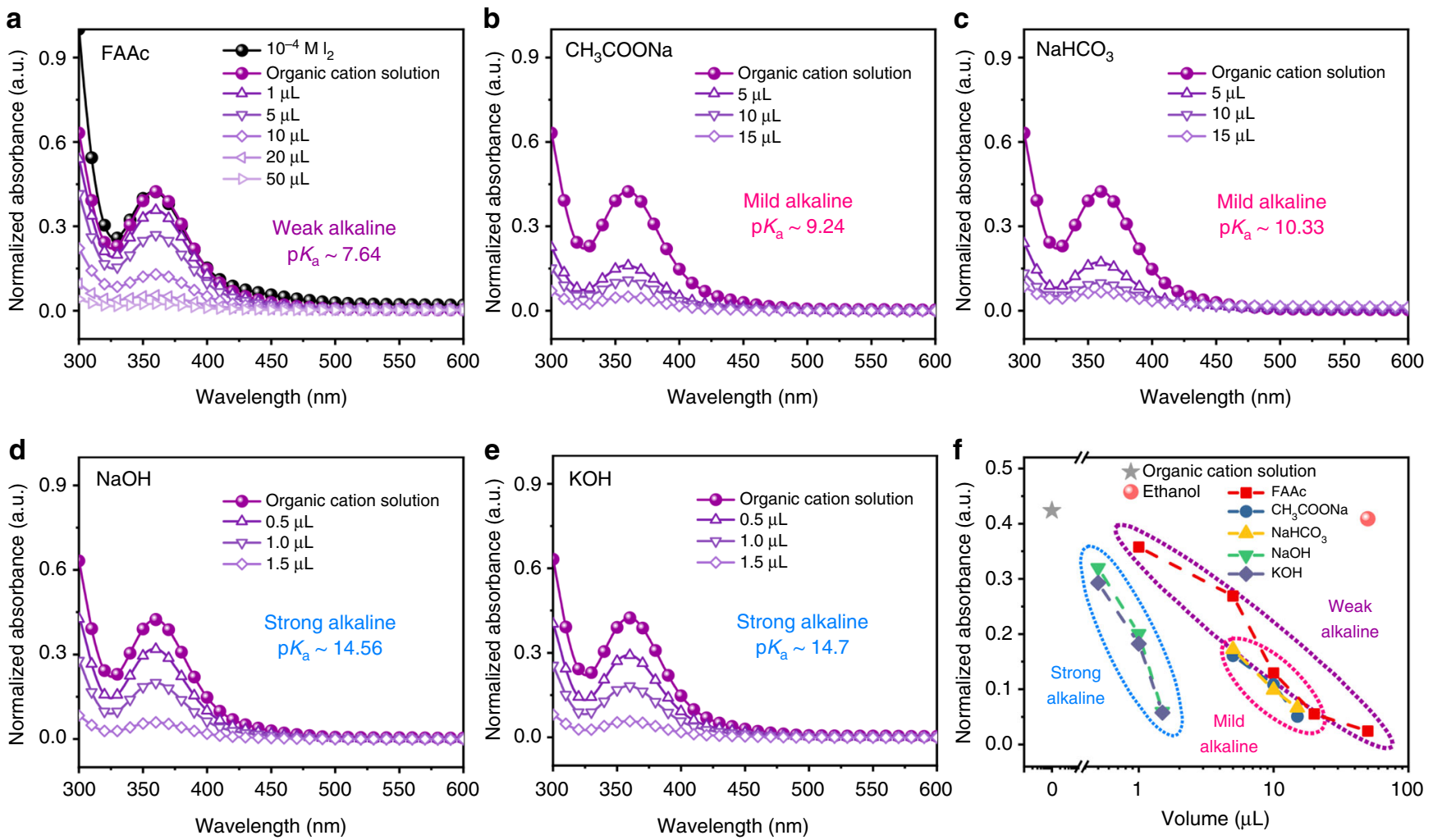

Fig. 1 Suppression of $\mathrm{I}_{2}$ impurity via alkaline additives. UV-vis absorption spectra of the organic cation solution, which is used to prepare the perovskite films, with different amounts of (a) FAAc, (b) $\mathrm{CH}_{3} \mathrm{COONa}$, (c) $\mathrm{NaHCO}_{3}$, (d) $\mathrm{NaOH}$, and (e) $\mathrm{KOH}$ ethanol solution. $\mathbf{f}$ Normalized absorbance for organic cation solution with different additives as a function of the volume

we performed scanning electron microscopy (SEM) and X-ray diffraction (XRD) measurements accordingly. Compared with the PVSK film, the perovskite films with $\mathrm{CH}_{3} \mathrm{COONa}$ or FAAc additive exhibited enlarged grain size (Fig. 2a-c), while those with $\mathrm{NaOH}$ or $\mathrm{KOH}$ remain the same grain size (Fig. 2d, Supplementary Fig. 3). It possibly indicates the different crystallization kinetics during film growth induced by the alkaline additives. Upon the employment of $\mathrm{CH}_{3} \mathrm{COONa}$ or FAAc, volatile acetate ions are reported to facilitate grain growth for better film quality ${ }^{33}$, which might account for the relatively enlarged grain size. On the contrary, perovskite films with the introduction of $\mathrm{NaOH}$ or $\mathrm{KOH}$ showed similar grain size, but a distinct crystalline grain morphology, as compared with the reference. It is often ascribed to the fact that $\mathrm{Na}^{+}$or $\mathrm{K}^{+}$is too small to incorporate into the perovskite lattice according to the Goldschmidt tolerance factor ${ }^{34}$. However, we cannot fully rule out the influences for crystal growth in the kinetics perspective, given the different chemical environments introduced by different alkalines, which will be discussed later.

Furthermore, XRD results showed a dominating $\alpha$-phase perovskite in all films (Fig. 2e), indicating that all these additives do not impact the thermodynamics of crystal growth substantially. However, $\mathrm{PbI}_{2}$ conversion process was affected by additives, as inferred from the formation of $\delta$-phase perovskite, as well as the residual $\mathrm{PbI}_{2}$. We purposely controlled the stoichiometry in all samples with a bit excessive of $\mathrm{PbI}_{2}$ for efficient passiva$\operatorname{tion}^{35,36}$ (see also Supplementary Note 4). We observed that perovskite films with mild alkaline additives $\left(\mathrm{CH}_{3} \mathrm{COONa}\right.$ or $\mathrm{NaHCO}_{3}$ ) only exhibited a relatively stronger $\mathrm{PbI}_{2}$ peak, while a distinct $\delta$-phase perovskite peak occurred when strong alkaline additives $(\mathrm{NaOH}$ or $\mathrm{KOH})$ were added during film fabrication. It suggests that the alkalinity of the additives would affect the formation of the by-products, where the stronger alkaline would promote the formation of the $\delta$-phase and suppress the formation of the $\alpha$-phase perovskite as well. To confirm our speculation, MA $\left(\mathrm{p} K_{a}=10.62\right) /$ ethanol was introduced into the precursor solution (Fig. 2f). We found a considerable amount of $\delta$-phase perovskite in the resultant perovskite film. As predicted, the formation of the $\alpha$-phase perovskite was suppressed substantially when the content of MA/ethanol was twice more. Interestingly, compared with the other additives, the weak alkaline FAAc resulted in the desirable phase quality, with significantly improved film crystallinity and less $\mathrm{PbI}_{2}$ content, as compared with that of the reference and other additives.

The intrinsic defects in perovskite films affect the photogenerated carrier behavior and device performance, which also would be affected by the alkalinity of the additives. We therefore conducted time-resolved photoluminescence (TRPL) analysis to study the dynamics of carrier recombination. The perovskite films were deposited on glass by using a precursor solution with different alkaline additives. The TRPL decay curves (Fig. 2g) were fitted with the bi-exponential rate law (Supplementary Table 1). We found that the perovskite films with addition of a weak alkaline possessed a longer carrier lifetime than those with the addition of a strong alkaline. The prolonged lifetime of perovskite films with a weak alkaline additive, compared with that of the PVSK film, was attributed to the suppression of incident $I_{2}$ in precursor solution and the desired film features, e.g., morphology and phase. Among all samples with alkaline additives, the perovskite film with FAAc exhibited the longest lifetime for both fast and slow recombination ( $\tau_{1}=132.7 \mathrm{~ns}$ and $\left.\tau_{2}=802.2 \mathrm{~ns}\right)$, indicating its lowest non-radiative recombination centers. To be noted, the introduction of $\mathrm{Na}^{+}$or $\mathrm{K}^{+}$also could possibly deform the perovskite crystal structure, which may lead to shallow defect sites or off-bandgap defects in perovskite films ${ }^{37-40}$ (see Supplementary Note 5). 

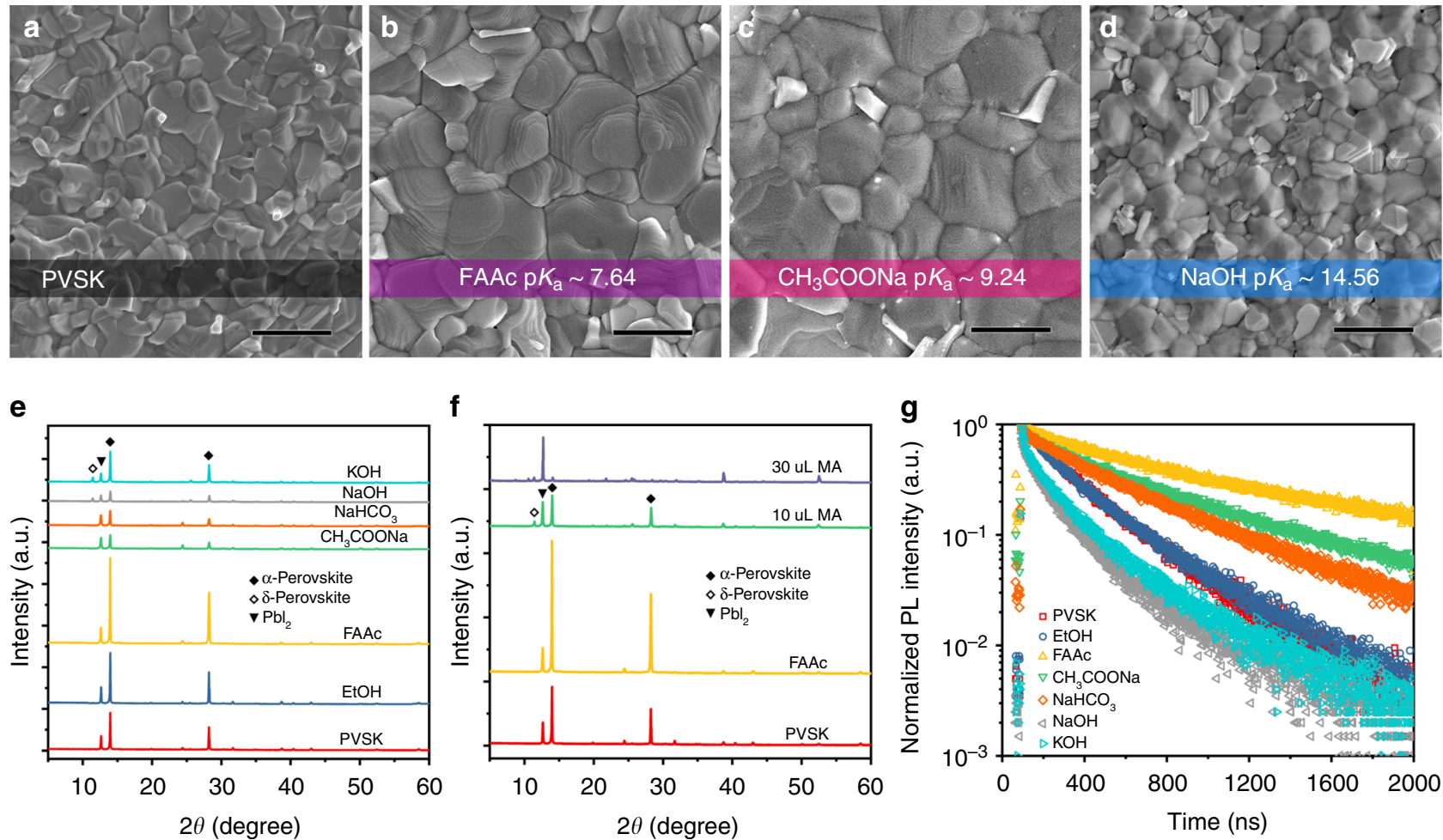

Fig. 2 Morphology, phase, and carrier lifetime analysis. SEM images for the perovskite films deposited on $\mathrm{SnO}_{2}$-coated ITO glass prepared from the precursor solution with (a) no additive, (b) FAAc, (c) $\mathrm{CH}_{3} \mathrm{COONa}$, and (d) $\mathrm{NaOH}$ ethanol solution, with a scale bar of $1 \mu \mathrm{m}$. e X-ray diffraction (XRD) pattern for the perovskite films deposited on $\mathrm{SnO}_{2}$-coated ITO glass prepared from the precursor solution with different alkaline additives. $\mathbf{f}$ XRD pattern for the perovskite films with NO additive, MA, and FAAc ethanol solution. $\mathbf{g}$ Time-resolved photoluminescence (TRPL) spectra for the perovskite films deposited on glass prepared from the precursor solution with different alkaline additives

Crystallization kinetics control via alkalinity. We further investigate the underlying crystallization kinetics of the formation of the $\alpha$-phase perovskite by developing an in situ UV-vis absorption measurement. In a typical experiment, the organic cation solution was spin-coated onto the annealed $\mathrm{PbI}_{2}$ layer, which was further characterized by a UV-vis absorption spectrometer immediately. By varying the alkalinity in precursor solution (Fig. 3a), we clearly observed that the formation of the $\alpha$ phase perovskite, whose main absorption peak ranged from 450 to $800 \mathrm{~nm}$, was suppressed substantially when the alkalinity gets stronger. To be noted, the absorption before $450 \mathrm{~nm}$ might stem from the $\mathrm{PbI}_{2}$ or the $\delta$-phase perovskite. Moreover, we judiciously selected two typical alkaline additives, e.g., FAAc (weak alkaline) and $\mathrm{NaOH}$ (strong alkaline), and continuously tracked the conversion evolution from $\mathrm{PbI}_{2}$ to the $\alpha$-phase perovskite. Compared with the reference (Fig. 3b), the weak alkaline does not affect the formation of the $\alpha$-phase perovskite, as expected. Accordingly, the absorption peak of the $\alpha$-phase perovskite in the main range $(450-800 \mathrm{~nm})$ is increased continuously as time grows. The inset figure in Fig. $3 \mathrm{~b}$ provides a visualization of the improved absorption in the range of 450 and $800 \mathrm{~nm}$, suggesting the continuous formation of the $a$-phase perovskite. On the contrary (Fig. 3c), the formation of the $\alpha$-phase perovskite with a strong alkaline was suppressed substantially. Even over time, only small changes occurred, as visualized in the inset figure of Fig. 3c. Based on the above analysis, we confirmed that the crystallization kinetics of the transformation from $\mathrm{PbI}_{2}$ to the a-phase perovskite, namely the activation energy, was influenced by the alkalinity of additives. As shown in scheme (Fig. 3d), when the alkalinity in the precursor gets stronger, the activation energy becomes larger, leading to an increased barrier for the transformation from the $\mathrm{PbI}_{2}$ to the $\alpha$-phase perovskite. The results suggest that while incident $\mathrm{I}_{2}$ could be effectively eliminated via a disproportionation reaction under various alkaline environments, the alkalinity of the additives is also crucial to fabricate highquality perovskite films with a desired phase. Because of the enrichment of the $\mathrm{OH}^{-}$anion when alkalinity gets stronger, we speculate that the oxygen in the $\mathrm{OH}^{-}$anion could participate in the coordination with lead iodide with stronger bonding. This may facilitate the structural transformation from $\mathrm{PbI}_{2}$ to the $\delta$ phase perovskite, leading to an increased energy barrier for the transformation of $\mathrm{PbI}_{2}$ to the $\alpha$-phase perovskite.

To verify the argument, we further performed the DFT simulation to analyze the exact activation energy for the formation of both the $a$-phase and $\delta$-phase of the $\mathrm{FAPbI}_{3}$ perovskite in different conditions. The Calculation setup was given in Methods and Supplementary Note 6. As shown in Fig. 3e, two converse trends of the activation energy change are observed upon the introduction of $\mathrm{OH}^{-}$into synthesis. At the low $\mathrm{OH}^{-}$concentration, the $\delta$-phase $\mathrm{FAPbI}_{3}$ has shown a much higher activation energy of $5.352 \mathrm{eV}$, which is $1.834 \mathrm{eV}$ higher than that of the $\alpha$-phase. As the $\mathrm{OH}^{-}$concentration increases, the activation energies of $\delta$-phase $\mathrm{FAPbI}_{3}$ decline from 5.352 to 4.143 $\mathrm{eV}$. However, the activation energies of $\alpha$-phase $\mathrm{FAPbI}_{3}$ increase from 3.518 to $4.293 \mathrm{eV}$, and finally exceed that of $\delta$-phase $\mathrm{FAPbI}_{3}$. It clearly suggests that the formation of the $\alpha$-phase perovskite is only preferred at a low $\mathrm{OH}^{-}$concentration. With the increase of $\mathrm{OH}^{-}$concentration, $\delta$-phase $\mathrm{FAPbI}_{3}$ is more preferred kinetically, which is in consistent with the observation of the experiments above.

Weak alkaline additives to reduce defect state density. To generalize the above findings, we selected three alkalines with a weak alkalinity, such as FAAc, triethanolamine hydrochloride 

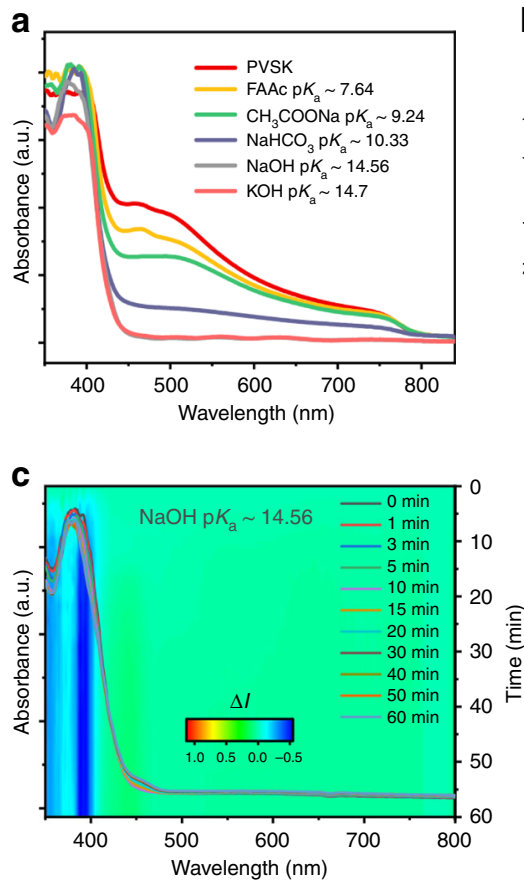
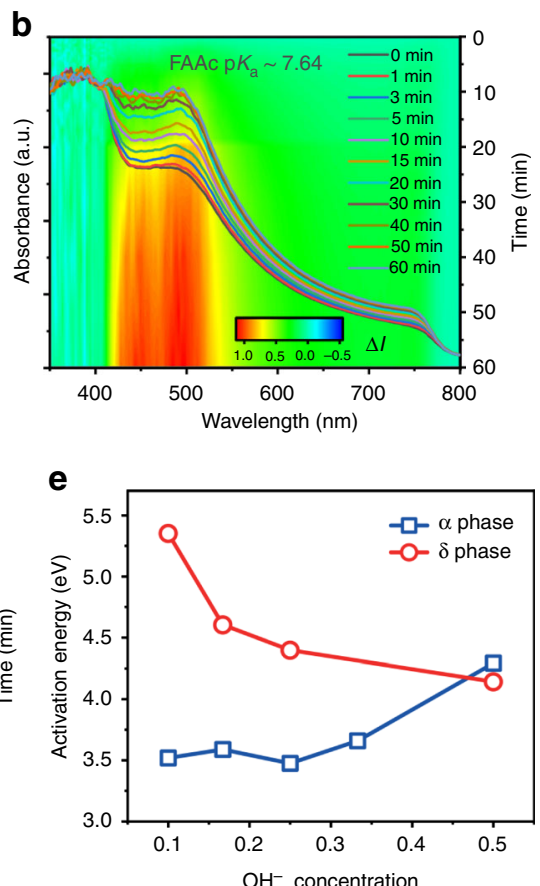

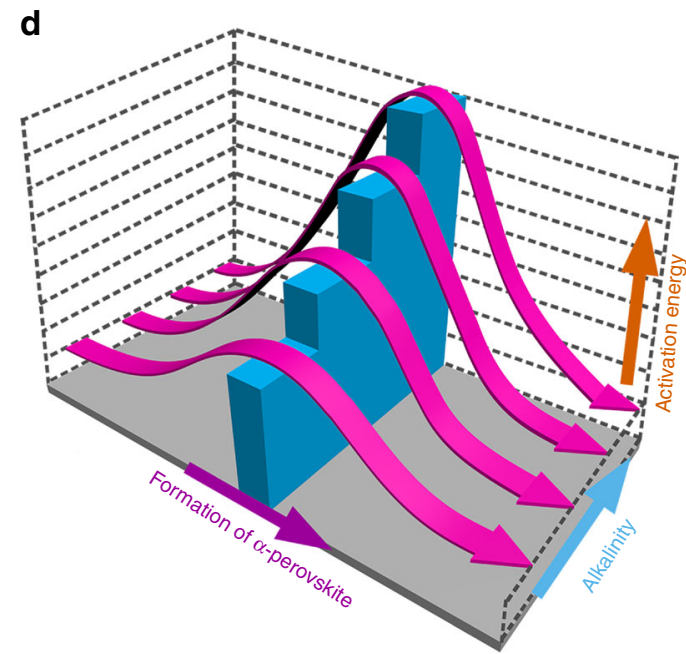

Fig. 3 Crystallization kinetics control of perovskite films. a Real-time UV-vis absorption spectra for the perovskite films without annealing prepared under

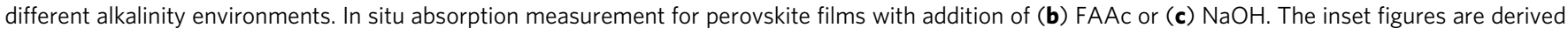
from the change of absorption intensity $(\Delta /)$ for each sample at different times and the $y$-axis is the right one (Time). $\mathbf{d}$ Schematic illustration for the impacts of alkalinity on the crystallization kinetics of perovskite films. e Activation energies of the $\alpha$ - and $\delta$-phase of FAPbl 3 in the dependence of $\mathrm{OH}^{-}$concentration

(TEAHCl, $\left.\mathrm{p} K_{a} \sim 7.8\right)$, and sodium hypophosphite $\left(\mathrm{NaH}_{2} \mathrm{PO}_{2}, \mathrm{p} K_{a}\right.$ $\sim 7.4$ ), and incorporated them into different perovskite material systems, respectively. We conducted the TRPL measurement to characterize the carrier recombination profile of different perovskite films. The corresponding devices are subject to photovoltaic performance investigations (see Supplementary Note 7). The results demonstrate that the additives with a weak alkalinity could successfully decrease the trap state density of iodine-based perovskite films via effectively inhibiting incident $I_{2}$ in the precursor solution. Combining the influence for alkalinity on the crystallization kinetics of perovskite films, the resultant devices show improved photovoltaic efficiency.

To obtain an in-depth understanding of the influence of a weak alkaline on the defect state of the perovskite, we chose the FAAc as an example and named the corresponding two-step processed perovskite film ((FA,MA,Cs) $\left.\mathrm{Pb}(\mathrm{I}, \mathrm{Br})_{3}-\mathrm{FAAc}\right)$ as the PVSK-FA. At first, steady-state photoluminescence (PL) spectra of PVSK and PVSK-FA over a temperature from 78 to $350 \mathrm{~K}$ were obtained and investigated (Fig. 4a, b). Within the temperature range of interest, the emission peak of PVSK-FA exhibited a distinct red shift as compared with PVSK, which was consistent with the UV-vis absorption results (Supplementary Fig. 4). It indicates a higher ratio of $\mathrm{FA}^{+}$incorporated into the PVSK-FA due to the addition of an additive in the precursor. It was documented that the $\mathrm{MAPbI}_{3}$ film exhibited a broadening inhomogeneous multi-peak emission feature from a lowtemperature phase, partially attributed to additional charge or exciton trap states only activated in low temperature ${ }^{41}$. However, in Figure $4 \mathrm{a}, \mathrm{b}$, we did not observe the above-mentioned multipeak feature at a low temperature in the PVSK and PVSK-FA, indicating the characteristics of less traps. With temperature increasing, we observed a broadening of the emission line due to the electron-phonon coupling near room temperature, which was a consistent recent study in the $\mathrm{FAPbI}_{3} \mathrm{film}^{42}$. Interestingly, the
PL spectra of the PVSK-FA exhibited a better symmetry than the PVSK with temperature increasing from 250 to $350 \mathrm{~K}$ (Fig. 4c). On the one hand, the absence of an asymmetric feature in this film may suggest significant reduction of defect states near the band edge. It is believed that reduced grain boundaries may lead to reduced defects ${ }^{43}$, which is consistent with the morphology analysis of larger grains in the corresponding films (Fig. 2a, b). On the other hand, better symmetry in the PL peak indicates the absence of shoulder peaks often attributed to the slight phase segregation in the mixed perovskites ${ }^{37}$. It is thus implied that PVSK-FA possibly exhibited a retarded phase segregation and decreased defect states, when compared with the PVSK.

We further carefully examined the luminescence efficiency of the absorber, which serves as an important measure to identify a high-quality semiconductor ${ }^{44-46}$. Generally, deep-level defects serve as non-radiative recombination centers to capture the photogenerated charge carriers, thereby dramatically cutting down the quantum efficiency of photoluminescence and photovoltaic efficiency in solar cells. To be noted, a good solar cell has to be a good light-emitting diode ${ }^{44}$. According to Shockley-Queisser equation, the $V_{\mathrm{OC}}$ of the device decreases when the luminescence quantum efficiency of the absorber decreases. Here, the PL quantum efficiency (PLQE) of the perovskite film was examined as a function of excitation power (Fig. 4d). The PLQE was relatively small for either PVSK or PVSK-FA at low excitation power, but rapidly went up with the increase of excitation power. It indicates that the radiative recombination prevails at high optical injection levels, wherein non-radiative recombination centers were filled up, which have been further discussed in Supplementary Note 8. Notably, the PLQE of PVSK-FA achieved $61 \%$ when the excitation power was $100 \mathrm{~mW} \mathrm{~cm}^{-2}$ (close to the device working condition), which is more than twice as that of PVSK (PLQE $=25 \%)$. It further revealed obvious enhancement of PLQE compared with PVSK 

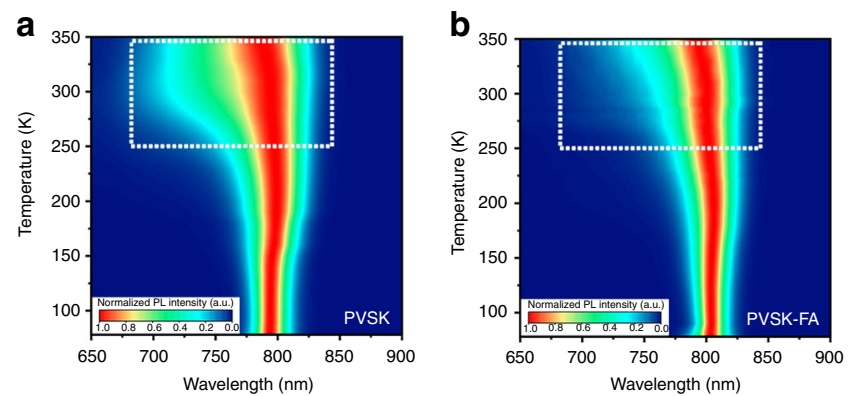

C

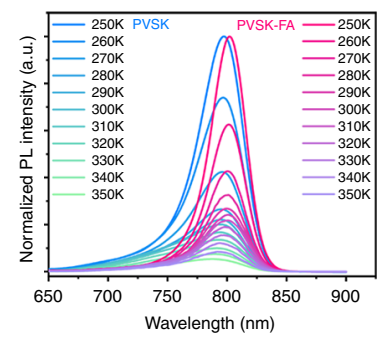

f

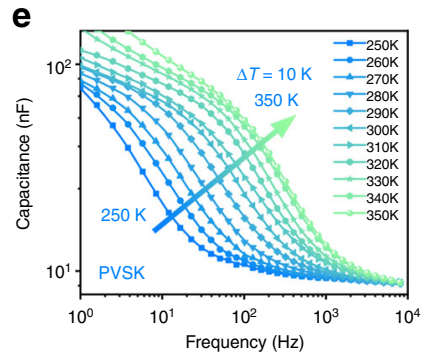

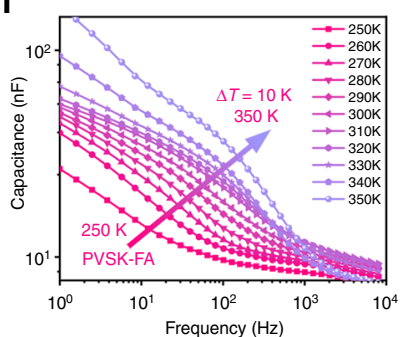

g

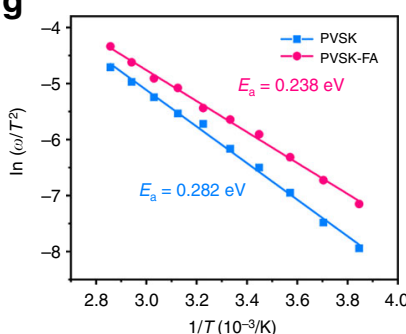

d

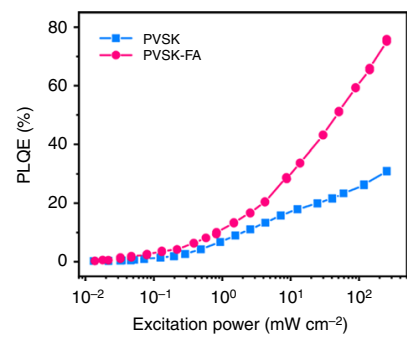

h

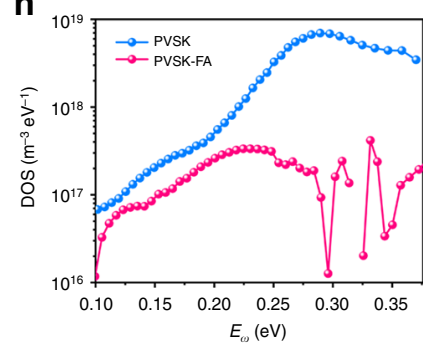

Fig. 4 Weak alkaline additives to reduce defect state density. Color plots of normalized steady-state PL spectra for (a) PVSK and (b) PVSK-FA at different temperatures from 78 to $350 \mathrm{~K}$. c Corresponding steady-state PL spectra for both films at various temperatures from 250 to $350 \mathrm{~K}$. d The PL quantum efficiency of PVSK and PVSK-FA films as a function of excitation power. The admittance spectra of the (e) PVSK and (f) PVSK-FA devices measured at a temperature from 250 to $350 \mathrm{~K}$ with a step of $10 \mathrm{~K}$. $\mathbf{g}$ The corresponding Arrhenius plots of the characteristic frequencies to extract the defect activation energy $\left(E_{\mathrm{a}}\right.$ ) for both devices. $\mathbf{h}$ Trap density of states (tDOS) for both devices obtained by thermal admittance spectroscopy at room temperature

among the entire excitation power range, confirming the reduction of deep-level defect state in PVSK-FA. It correlates to the improvement in $V_{\mathrm{OC}}$ in the corresponding device, as will be discussed later.

To find the defect energy level in the perovskite films, we conducted thermal admittance spectroscopy (TAS) analysis. It is widely adopted in perovskite solar cells to extract the native defect state-level information through the identification of junction capacitance. Given a p-type perovskite semiconductor, the defect activation energy $\left(E_{\mathrm{a}}\right)$ is approximately the depth of defect state energy level relative to the valance band of the perovskite ${ }^{47,48}$. Here, TAS measurement was carried out from 250 to $350 \mathrm{~K}$ with a step of $10 \mathrm{~K}$ to obtain the temperature-dependent admittance spectra for PVSK and PVSK-FA-based devices (Fig. 4e, f). The characteristic transition angular frequency $\left(\omega_{0}\right)$ was extracted from the derivative of the capacitance-frequency spectrum. The relationship between $E_{\mathrm{a}}$ and $\omega_{0}$ follows the equation: $\omega_{0}=\beta T^{2}$ $\exp \left(-E_{\mathrm{a}} / k T\right)$, where $\beta$ is a temperature-dependent parameter, $k$ is the Boltzmann's constant, and $T$ is the temperature. The Arrhenius plot (Fig. $4 \mathrm{~g}$ ) derived from the temperature-dependent $\omega_{0}$ and fitted curves revealed that the $E_{\mathrm{a}}$ of PVSK and PVSK-FA devices were 0.282 and $0.238 \mathrm{eV}$, respectively.

Moreover, we attempted to illustrate the energetic profile regarding the trap density of states (tDOS) in the perovskite absorber (Fig. 4h). It could be derived from the equation $N_{\mathrm{T}}\left(E_{\omega}\right)=-\omega V_{\mathrm{bi}} / q k T W \times \mathrm{d} C / \mathrm{d} \omega$ and $E_{\omega}=k T \ln \left(\beta T^{2} / \omega\right)$, where $q$ is the elementary charge, $\omega$ is the angular frequency, $V_{\mathrm{bi}}$ is the built-in potential, and $W$ is the depletion region, respectively ${ }^{49}$. The $V_{\mathrm{bi}}$ and $W$ were obtained from the Mott-Schottky analysis (Supplementary Fig. 5). As expected, the PVSK device exhibited a relatively large density of defect states, especially around the deep defect level at $0.282 \mathrm{eV}$. Previous DFT calculation study ${ }^{26}$ reported that this defect-state level $(0.282 \mathrm{eV})$ could potentially be ascribed to iodine interstitials $\left(\mathrm{I}_{\mathrm{i}}\right)$, which was the deep-level defect state and dominating non-radiative recombination centers in devices. Interestingly, the PVSK-FA device exhibited a striking reduction of the deep-level defect state at $0.282 \mathrm{eV}$ for two orders of magnitude. In addition, the shallow defect states $(0.1-0.2 \mathrm{eV})$ were also suppressed. Considering the analysis above, we reasonably claim that the introduction of a weak alkaline additive could effectively suppress the incident $I_{2}$ in the precursor solution and significantly reduce the $I_{i}$ defect states and achieve a much smaller defect activation energy in the resultant perovskite films. In this case, our iodine suppression approach looks contradictory with the previous research ${ }^{29}$; however, we argue that the discrepancy in these two approaches of iodine management is mostly due to different physiochemical properties in the corresponding absorber layers. It likely stemmed from the two film growth techniques applied and different material compositions, which is further discussed in Supplementary Note 9.

Beside of defects at the deep energy level, tail defects at the band edge were further investigated. Urbach tail profiles of perovskite films were calculated according to the formula equation: $\alpha=\alpha_{0} \exp \left(h v / E_{\mathrm{U}}\right)$, where $\alpha_{0}$ is a constant, $E_{\mathrm{U}}$ denotes Urbach energy, and $\alpha$ is an absorption coefficient. The absorption coefficient versus energy plots were presented in Supplementary Fig. 6. As is reported 33,50 , low $E_{U}$ is highly desirable for semiconductor devices, indicative of less electronic disorders in the crystal lattice (or less impurities). Accordingly, the $E_{\mathrm{U}}$ values were estimated to be 31.22 and $29.64 \mathrm{meV}$ for PVSK and PVSK-FA, respectively. Assuming the same level of thermal disorder, the smaller $E_{\mathrm{U}}$ value obtained from PVSK-FA demonstrated a lower level of electronic disorder at the band edge.

To quantitatively assess the concentration of defects across the entire device, the current density-voltage curves under the dark state for capacitor-like devices with indium tin oxide (ITO)/ perovskite/gold $\mathrm{Au}$ ) configuration were measured (Supplementary Fig. 7). The linear current density-voltage relation at the low bias suggested an ohmic response, and the transition point was considered as the voltage at which all the traps were filled (trapfilled limit voltage: $\left.V_{\mathrm{TFL}}\right)$. The concentration of defects was determined by the equation $N_{\text {defects }}=2 \varepsilon \varepsilon_{0} V_{\mathrm{TFL}} / q L^{2}$, where $q$ is the elementary charge, $\varepsilon$ is the relative dielectric constant, $\varepsilon_{0}$ is the 

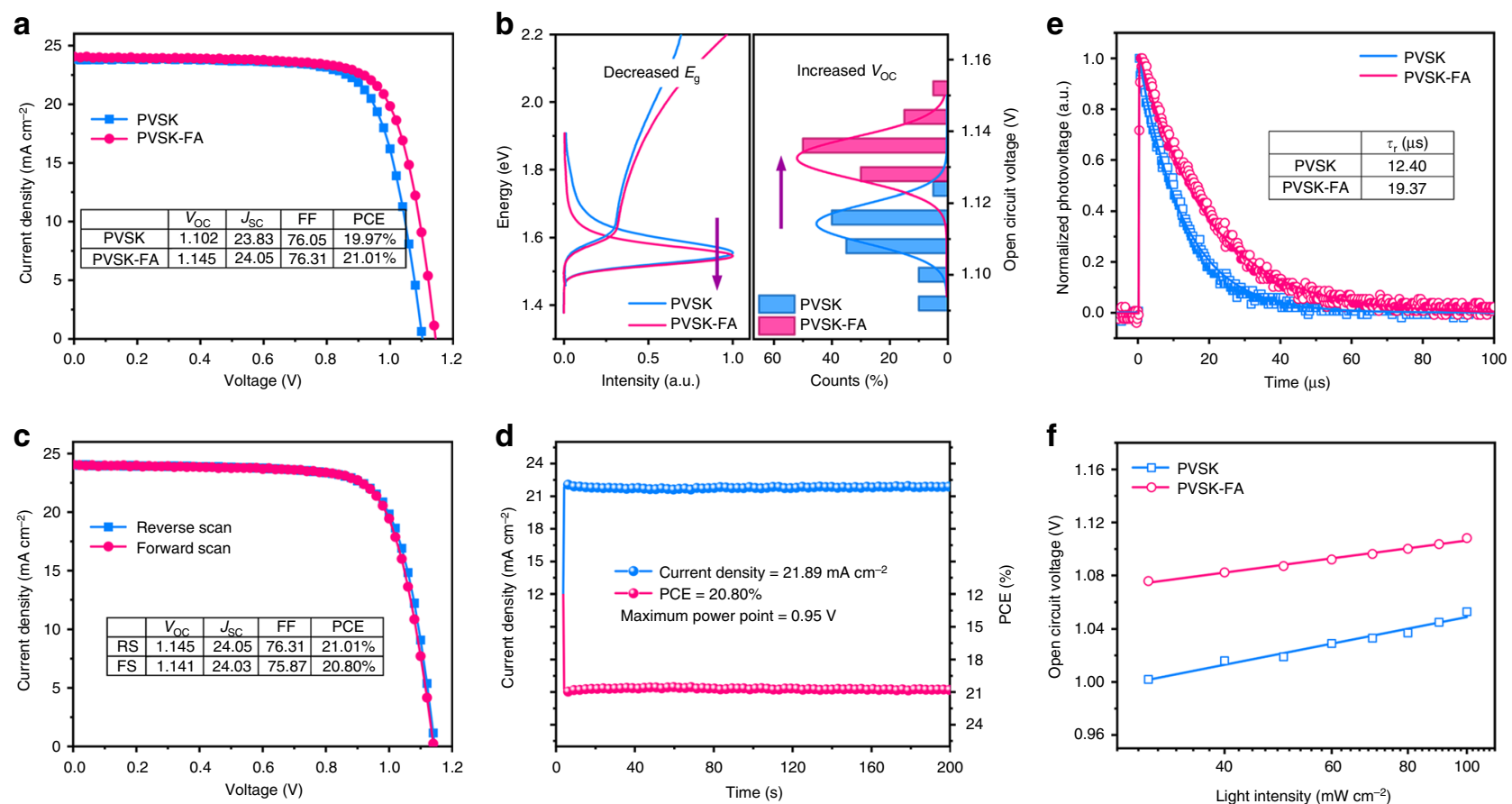

Fig. 5 Photovoltaic device performance. a Current density-voltage curves for PVSK and PVSK-FA devices. b Left part: the UV-vis and PL spectra of for the PVSK and PVSK-FA films. Right part: the histogram of $V_{O C}$ for the PVSK and PVSK-FA devices and the curve represents Gaussian function fit to the statistics data. c The champion device performance under reverse scan (1.2 to $-0.1 \mathrm{~V})$ and forward scan ( -0.1 to $1.2 \mathrm{~V})$, with a scan rate of $40 \mathrm{mV} \mathrm{s}{ }^{-1}$. d Steady-state current density and power conversion efficiency holding the voltage at the maximum power point (0.95 V) for the PVSK-FA device. All these photovoltage characterizations were carried out under standard AM 1.5 radiation at ambient condition. e The transient photovoltage decay curves and (f) $V_{O C}$ as a function of light intensity for the PVSK and PVSK-FA devices

vacuum permittivity, and $L$ is the thickness of the perovskite films obtained from the cross-sectional SEM images of the ITO/ perovskite/Au devices (Supplementary Fig. 8). It is reasonable to assume that the slight introduction of an additive would not affect the relative dielectric constants of perovskite films significantly ${ }^{51}$. The defect density $N_{\text {defects }}$ was estimated to be $1.38 \times 10^{16}$ and $0.47 \times 10^{16} \mathrm{~cm}^{-3}$ for PVSK and PVSK-FA devices, respectively. It clearly confirms that the defect density in the perovskite absorber has been significantly reduced to nearly an order of magnitude by the addition of a weak alkaline additive. Moreover, we have conducted femtosecond optical measurements ${ }^{52}$ and confirmed that PVSK-FA exhibited a lower defect density than the PVSK film, which is the same order of magnitude as the above result (Supplementary Note 10).

Weak alkaline additive to improve photovoltaic performance. The significant reduction in intrinsic defect density within the perovskite film was expected to suppress the non-radiative recombination channel effectively and improve the device performance. To prove it, we fabricated the planar heterojunction devices using the structure $\mathrm{ITO} / \mathrm{SnO}_{2} /$ perovskite/spiro-OMe$\mathrm{TAD} / \mathrm{Au}$ (Supplementary Fig. 9) and studied the impacts of weak alkaline additives on photovoltaic performance. Figure 5a presents the current density-voltage $(J-V)$ curves of the optimized perovskite devices measured under standard AM $1.5 \mathrm{G}$ at ambient condition. The typical PVSK device yielded a PCE of $19.97 \%$ with a $V_{\mathrm{OC}}$ of $1.102 \mathrm{~V}$, a short-circuit current density $\left(J_{\mathrm{SC}}\right)$ of 23.83 $\mathrm{mA} \mathrm{cm}{ }^{-2}$, and a fill factor (FF) of $76.05 \%$. Meanwhile, the best PVSK-FA device exhibited a significantly improved performance with a $V_{\mathrm{OC}}$ of $1.145 \mathrm{~V}$, a $J_{\mathrm{SC}}$ of $24.05 \mathrm{~mA} \mathrm{~cm}^{-2}$, a FF of $76.31 \%$, and the corresponding PCE of $21.01 \%$. One of our best devices was sent to the accredited PV calibration laboratory (Newport,
USA) and the certified PCE was 20.87\% (Supplementary Fig. 10). The slightly enhanced $J_{\mathrm{SC}}$ value was attributed to improved lightharvesting capacity and a slightly red shift of the absorption onset. The major improvement in device performance was contributed from $V_{\mathrm{OC}}$ enhancement, thanks to the high-quality perovskite thin film with significantly reduced intrinsic defect density by the addition of a weak alkaline additive.

To be emphasized, in the present study, the addition of FAAc could slightly reduce the bandgap of the resultant film from $1.57 \mathrm{eV}$ to $1.56 \mathrm{eV}$ but increase $V_{\mathrm{OC}}$ from 1.102 to $1.147 \mathrm{~V}$ in the resulting device. It is interesting that although the bandgap of the perovskite film decreased, the $V_{\mathrm{OC}}$ of the relevant solar devices actually improved (Fig. 5b). It thus reveals one vital feature for this high-efficiency device, which is the small $V_{\mathrm{OC}}$ deficit (defined as $E_{\mathrm{g}} / q-V_{\mathrm{OC}}$, where $E_{\mathrm{g}}$ is the optical bandgap and $q$ is the elemental charge). $V_{\mathrm{OC}}$ deficit is an essential indicator to measure the potential of the photovoltaic material and the maturity of the relevant technology. Current $V_{\mathrm{OC}}$ deficit for c-silicon, GaAs, and organic solar cells are around 400,300 , and $600 \mathrm{mV}$, respectively ${ }^{53}$. Surprisingly, the corresponding $V_{\mathrm{OC}}$ deficit in our device decreased to $413 \mathrm{mV}$ upon FAAc addition, wherein both the $V_{\mathrm{OC}}$ $(1.147 \mathrm{~V})$ and the bandgap $(1.56 \mathrm{eV})$ were certified through a third party. The $V_{\mathrm{OC}}$ deficit of $413 \mathrm{mV}$ is one of the smallest certified value for planar heterojunction perovskite solar cells to date. It further implies substantially reduced non-radiative recombination losses in the PVSK-FA device, which was consistent with the high PLQE measured previously.

In addition, we did not observe an appreciable $J-V$ hysteresis in the corresponding device, and the average PCE of $20.91 \%$ was achieved (Fig. 5c and Supplementary Fig. 10). The integrated photocurrent density gave a calculated value of $23.47 \mathrm{~mA} \mathrm{~cm}^{-2}$ from the external quantum efficiency (EQE) spectrum (Supplementary Fig. 11), which was comparable with the result from $J-V$ 
measurement. In addition, a steady-state current density of $21.89 \mathrm{~mA} \mathrm{~cm}^{-2}$ and a stabilized PCE of $20.80 \%$, were also achieved by holding the voltage at the maximum power point $(0.95 \mathrm{~V})$ for the PVSK-FA device (Fig. 5d). To probe the reliability and repeatability, 20 individual devices were fabricated with the identical processing condition and the corresponding statistical distribution of the PCE values was presented (Supplementary Fig. 12). The average efficiency for the PVSK and PVSK-FA devices were $19.34 \%$ and $20.63 \%$, respectively. Furthermore, we have investigated the long-term stability of the PVSK and PVSK-FA-based devices, as shown in Supplementary Fig. 13. The reference without further encapsulation degraded to be around $80 \%$ within $1500 \mathrm{~h}$ of storage in an $\mathrm{N}_{2}$ glove box, whereas the PVSK-FA-based devices exhibited less degradation. It is possibly because of the higher thin-film quality of PVSK-FA with reduced defect states, which inhibits the penetration of undesirable water or oxygen molecules that further decompose perovskite films. Meanwhile, it may also correlate to the elimination of incident $\mathrm{I}_{2}$, which has been demonstrated to induce the chemical chain degradation of the perovskite ${ }^{31}$.

To gain further insights into the carrier dynamics across the device, we measured transient photocurrent (TPC) and photovoltage (TPV) decays of PVSK and PVSK-FA-based devices on the microsecond scale ${ }^{54}$. The PVSK-FA-based device exhibited a faster decay than that of the PVSK-based device, and the charge transport lifetime $\left(\tau_{\mathrm{t}}\right)$ derived from TPC measurements (Supplementary Fig. 14) was decreased from 4.13 to $1.97 \mu$ s, indicating a dramatic improvement of charge transport capability for the PVSK-FA-based device. The TPV curves were correlated to the carrier recombination rates under the open-circuit condition in the full cell. We observed that the PVSK-FA-based device demonstrated a much slower decay, when compared with that of the PVSK-based device (Fig. 5e). The charge recombination lifetime $\left(\tau_{\mathrm{r}}\right)$ derived from the TPV curves was significantly prolonged from 12.40 to $19.37 \mu$ s, suggesting that the carrier recombination was predominately prohibited in the device. It indicates that the prolonged lifetime constant $\tau_{\mathrm{r}}$ contributed from substantially reduced defect density in the perovskite material by the addition of weak alkaline additives.

Furthermore, we measured the $V_{\mathrm{OC}}$ change as a function of illumination intensity to elucidate the carrier recombination mechanism during device operation. For both PVSK and PVSK-FA-based devices, the $V_{\mathrm{OC}}$ increased monotonically with incident light intensity (Fig. 5f). According to the equation $V_{\mathrm{OC}}=n k T / q \ln \left(I_{\mathrm{SC}} / I_{0}+1\right)$, the values of the ideality factor were calculated to be 1.51 and 1.02 , respectively. Generally, the ideality factor derails from unity if Shockley-Read-Hall (SRH) recombination occurs ${ }^{55}$. In our study, the ideality factor for the PVSK-FA-based device showed a significant reduction from 1.51 to 1.02 , when compared with that of the PVSK-based device. It demonstrates that trap-assisted SRH recombination was suppressed substantially due to the reduced intrinsic defect density, which was in good agreement with previous results.

\section{Discussion}

In summary, we demonstrate a universal pathway to effectively suppress the incident $\mathrm{I}_{2}$ defects and manipulate the stoichiometry via the iodine disproportionation reaction under an alkaline environment. The alkalinity is proven to be a crucial parameter that greatly impacts the crystallization kinetics and optoelectronic properties of the perovskite films. A weak alkaline consisting of the $\mathrm{FA}^{+}$cation was demonstrated to serve as a 'residual' free agent to suppress deep-level defect density in the mixed perovskites ((FA,MA,Cs) $\left.\mathrm{Pb}(\mathrm{I}, \mathrm{Br})_{3}\right)$, which provides extra benefits in tuning the A-site stoichiometry and film morphology. Featured with a narrower bandgap of the absorber though, the device exhibited a significant enhancement on $V_{\text {OC. }}$. It observed one of the smallest $V_{\text {OC }}$ deficits of $413 \mathrm{mV}$ in a certified planar heterojunction perovskite solar cell with an average PCE of $20.87 \%$. These findings would bridge the gap between precursor chemistry and deep-level defect behavior in the perovskite film, particularly the iodide-based perovskite, which guides the design of nextgeneration perovskite solar cells with an efficiency approaching the theoretical limit.

\section{Methods}

Materials. All the reagents and chemicals were used as received, including $\mathrm{PbI}_{2}$ (99.999\%, Sigma Aldrich), CsI (99.90\%, Aladdin Industrial Corporation), N,Ndimethylformamide (99.99\%, Sigma Aldrich), dimethyl sulfoxide ( $99.50 \%$, Sigma Aldrich), sodium acetate (AR, Aladdin Industrial Corporation), formamidine acetate (99\%, Aladdin Industrial Corporation), sodium bicarbonate (AR, Aladdin Industrial Corporation), sodium hydroxide (AR, Aladdin Industrial Corporation), potassium hydroxide (AR, Aladdin Industrial Corporation), sodium hypophosphite (99\%, Aladdin Industrial Corporation), triethanolamine hydrochloride (99.5\%, Aladdin Industrial Corporation), spiro-OMeTAD (Xi'an Polymer Light Technology Corp.), chlorobenzene (99.9\%, Aladdin Industrial Corporation), 4tertbutylpyridine (99.90\%, Sigma Aldrich), lithium bis(trifluoromethylsulfonyl) imide (99.95\%, Sigma Aldrich), anhydrous ethyl alcohol (99.5\%, Sigma Aldrich, product code: 459836), anhydrous isopropanol (99.5\%, Sigma Aldrich, product code: 278475), and tin-doped indium oxide (ITO) substrates. All the formamidinium iodide (FAI), methylammonium iodide (MAI), methylammonium bromine (MABr), and methylammonium chloride (MACl) were synthesized and purified according to the procedure mentioned in the pervious literature ${ }^{54}$.

Preparation of the $\mathrm{SnO}_{\mathbf{2}}$ films. The tin-doped indium oxide (ITO) substrates were cleaned with acetone, deionized water, and isopropanol ultrasonically. The tin (IV) oxide colloidal dispersion $\left(\mathrm{SnO}_{2}, 15 \%\right.$ in $\mathrm{H}_{2} \mathrm{O}$ colloidal dispersion) was purchased from Alfa Aesar. The precursor solution was diluted by $\mathrm{H}_{2} \mathrm{O}$ to $2.67 \%$ and then spin-coated onto glass/ITO substrates at $4000 \mathrm{rpm}$ for $30 \mathrm{~s}$, and finally baked on a hot plate at $150^{\circ} \mathrm{C}$ for $30 \mathrm{~min}$ in ambient air.

Preparation of the perovskite precursor and films. To prepare the $\mathrm{PbI}_{2}$ and organic cation precursors, $600 \mathrm{mg}$ of $\mathrm{PbI}_{2}$ and $18 \mathrm{mg}$ of CsI were dissolved in $1 \mathrm{~mL}$ of mixture solvent of DMF:DMSO (9:1 v:v) and then annealed on a hot plate at $70{ }^{\circ} \mathrm{C}$ for $2 \mathrm{~h}$ with vigorous stirring. In all, $40 \mathrm{mg}$ of FAI, $19 \mathrm{mg}$ of MAI, $6 \mathrm{mg}$ of $\mathrm{MABr}$, and $5 \mathrm{mg}$ of $\mathrm{MACl}$ were dissolved in $1 \mathrm{~mL}$ of isopropanol and then continuously stirred for $2 \mathrm{~h}$ at room temperature. To prepare the alkaline additive solution, $45 \mathrm{mg}$ of different additives were dissolved in $1 \mathrm{~mL}$ of anhydrous ethyl alcohol at $70^{\circ} \mathrm{C}$ for $30 \mathrm{~min}$ with vigorous stirring. Due to the different solubility of alkaline additives in ethyl alcohol, the possible supernatant was separated (Supplementary Note 11). For the certified perovskite solar cell, $45 \mathrm{mg}$ of FAAc were dissolved in $1 \mathrm{~mL}$ of ethyl alcohol at $70^{\circ} \mathrm{C}$ for $30 \mathrm{~min}$ with vigorous stirring. Then, $30 \mu \mathrm{L}$ of FAAc ethanol solution was added to $1 \mathrm{~mL}$ of organic cation solution before being prepared with the perovskite film. The typical sequent-step method was employed to fabricate the perovskite film. First, the $\mathrm{PbI}_{2}$ precursor was spincoated on the $\mathrm{SnO}_{2}$ substrate in an $\mathrm{N}_{2}$ glove box at $2300 \mathrm{rpm}$ for $30 \mathrm{~s}$ (accelerated speed $6000 \mathrm{rpm} \mathrm{s}^{-1}$ ) and then annealed at $70^{\circ} \mathrm{C}$ for 1 min in an $\mathrm{N}_{2}$ glove box. Then, the organic cation precursor was spin-coated on the $\mathrm{PbI}_{2}$ film at $2000 \mathrm{rpm}$ for $30 \mathrm{~s}$ (accelerated speed $6000 \mathrm{rpm} \mathrm{s}^{-1}$ ) in an $\mathrm{N}_{2}$ glove box. Finally, the film was kept under vacuum for $5 \mathrm{~min}$ and annealed at $150{ }^{\circ} \mathrm{C}$ for $15 \mathrm{~min}$ in ambient air to form the perovskite film.

\section{Preparation of the Spiro-OMeTAD films and gold electrode. The Spiro-}

OMeTAD solution was first prepared by dissolving $72.3 \mathrm{mg}$ of Spiro-OMeTAD, $17.5 \mu \mathrm{L}$ of a stock solution of $520 \mathrm{mg} \mathrm{mL}^{-1} \mathrm{LiTFSI} /$ acetonitrile, and $28.8 \mu \mathrm{L}$ of tBP in $1 \mathrm{~mL}$ of chlorobenzene. Then the Spiro-OMeTAD precursor was spin-coated at $3000 \mathrm{rpm}$ for $30 \mathrm{~s}$ (accelerated speed $6000 \mathrm{rpm} \mathrm{s}^{-1}$ ) in an $\mathrm{N}_{2}$ glove box. Finally, $100-\mathrm{nm}$-thick gold was thermally evaporated as a counterelectrode under a pressure of $1 \times 10^{-4} \mathrm{~Pa}$ on top of the Spiro-OMeTAD films to form the back contact by using a shadow mask to pattern the electrode.

Calculation setup. The calculations have been applied through DFT within CASTEP packages ${ }^{56}$. For the functional, the Perdew-Burke-Ernzerhof-based generalized gradient approximation (GGA) ${ }^{57}$ has been selected to describe the electronic exchange-correlation interaction. All the geometry optimizations have been operated based on the Broyden-Fletcher-Goldfarb-Shannon (BFGS) algorithm, in which the residue of the Hellmann-Feynman forces will be converged to less than $0.001 \mathrm{eV} \mathrm{A}^{-1}$. The additional convergence criteria for the total energy and the interionic displacement are set to be less than $5 \times 10^{-5} \mathrm{eV}$ per atom and $0.005 \AA$ per atom, respectively. The ultrafine plane-wave cutoff energy with the ultrasoft pseudopotential scheme has been chosen as the basis set. The $\mathrm{OH}^{-}$ 
molecule has been inserted into the $\mathrm{PbI}_{2}$ model and $\mathrm{FAPbI}_{3}$ unit cells with a different ratio $\mathrm{n}(n=0.1-0.5)$ to represent the different concentration, which can be referred to as '\#/unit cell' in this simulation. The corresponding effect to the activation energies will be calculated based on the following equation:

$$
E_{\mathrm{act}}=E_{\mathrm{FAPbI}_{3(\mathrm{OH}) \mathrm{n}}}-E_{\mathrm{PbI}_{2(\mathrm{OH}) \mathrm{n}}}-E_{\mathrm{FAI}}
$$

The Monkhost-Pack reciprocal space integration was performed using a spacing of $0.07 \AA^{-1}$ in all models with a different $\mathrm{OH}^{-}$concentration ${ }^{58}$, which was also self-consistently suggested by total energy minimization. With this special $\mathrm{k}$ point sampling, the total energy is converged within the maximum expected selfconsistent cycles.

Characterization. The absorbance of different precursor solutions and perovskite films was measured by a Hitachi UH4150 spectrophotometer. The integrating sphere was employed when we characterized the perovskite film. A field-emission SEM instrument (Hitachi S-4800) was used to acquire SEM images. The electron beam was accelerated at $5.0 \mathrm{kV}$. Temperature-dependent steady-state photoluminescence (PL) spectra were obtained by FLS980 (Edinburgh Instruments Ltd.), equipped with a Xe lamp, a liquid nitrogen cryostat (Oxford Instruments, OptistatDN-V), and a photomultiplier tube (PMT) detector. The excitation wavelength was $470 \mathrm{~nm}$. The picosecond pulsed diode laser (EPL-470, Edinburgh Instruments Ltd.) was used to measure PL lifetime with a repetition rate of $0.2 \mathrm{MHz}$, a pulse

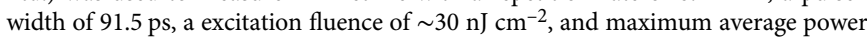
of $5 \mathrm{~mW}$. The PLQE data were obtained from a three-step technique through the combination of a 445-nm continuous-wave laser, a spectrometer, an optical fiber, and an integrating sphere ${ }^{59,60}$. The powder XRD spectra $(\theta-2 \theta$ scans) were taken on a X'Pert3 powder X-ray diffraction system (PANalytical Inc.) with $\mathrm{Cu} \mathrm{Ka}$ $(\lambda=1.5418 \AA)$ as the $\mathrm{X}$-ray source. The thermal admittance spectroscopy were conducted on Zahner Zennium pro Electrochemical Workstation at various temperatures $(T=250-350 \mathrm{~K})$ in the dark from $10^{0}$ to $10^{6} \mathrm{~Hz}$. A small AC voltage of $50 \mathrm{mV}$ was used, and the DC bias was kept at zero to avoid the influence of the ferroelectric effect for perovskite materials during measurement. For temperaturedependent characterization, the sample was mounted in the Cryo Industries Liquid Nitrogen Dewars with Lake Shore model 335 cryogenic temperature controller. The current density-voltage $(J-V)$ curves were measured (2400 Series SourceMeter, Keithley Instruments) under a simulated AM $1.5 \mathrm{G}$ illumination at $100 \mathrm{~mW} \mathrm{~cm}^{-2}$ (Newport Thermal Oriel 91192 1000-W solar simulator). The light intensity was calibrated using a KG-5 Si diode. The $J-V$ measurements were carried out in ambient air. The sweep speed was fixed at $40 \mathrm{mV} \mathrm{s}^{-1}$ (step $0.02 \mathrm{~V}$, delay time $500 \mathrm{~ms}$ ). The shading mask and one of our best device were sent to an accredited PV calibration laboratory (Newport, USA) for certification. The active area was defined as $0.09408 \mathrm{~cm}^{2}$. External quantum efficiencies (EQE) were obtained by an Enli Technology (Taiwan) EQE measurement system. A calibrated silicon diode with the known spectral response was used as a reference. For the transient photovoltage or photocurrent (TPV or TPC) decay measurements, a white light bias was from an array of diodes (Molex 180081-4320) to simulate 1-sun working condition, and the carriers were excited by a $532-\mathrm{nm}$ pulse laser. The dynamics curves were recorded on a digital oscilloscope at a mega $\Omega$ (open-circuit condition for TPV) and a $50-\Omega$ (short-circuit condition for TPC) resistor.

\section{Data availability}

The data that support the findings of this study are available from the corresponding author upon request.

Received: 11 August 2018 Accepted: 16 February 2019

Published online: 07 March 2019

\section{References}

1. Park, N.-G., Grätzel, M., Miyasaka, T., Zhu, K. \& Emery, K. Towards stable and commercially available perovskite solar cells. Nat. Energy 1, 16152 (2016).

2. Yin, W. J., Shi, T. \& Yan, Y. Unique properties of halide perovskites as possible origins of the superior solar cell performance. Adv. Mater. 26, 4653-4658 (2014).

3. Yin, W. J., Shi, T. \& Yan, Y. Unusual defect physics in $\mathrm{CH}_{3} \mathrm{NH}_{3} \mathrm{PbI}_{3}$ perovskite solar cell absorber. Appl. Phys. Lett. 104, 063903 (2014).

4. Buin, A., Comin, R., Xu, J., Ip, A. H. \& Sargent, E. H. Halide-dependent electronic structure of organolead perovskite materials. Chem. Mater. 27, 4405-4412 (2015).

5. Shi, D. et al. Low trap-state density and long carrier diffusion in organolead trihalide perovskite single crystals. Science 347, 519-522 (2015)

6. Kojima, A., Teshima, K., Shirai, Y. \& Miyasaka, T. Organometal halide perovskites as visible-light sensitizers for photovoltaic cells. J. Am. Chem. Soc. 131, 6050-6051 (2009)
7. Im, J. H., Jang, I. H., Pellet, N., Grätzel, M. \& Park, N. G. Growth of $\mathrm{CH}_{3} \mathrm{NH}_{3} \mathrm{PbI}_{3}$ cuboids with controlled size for high-efficiency perovskite solar cells. Nat. Nanotech. 9, 927-932 (2014).

8. Saliba, M. et al. Incorporation of rubidium cations into perovskite solar cells improves photovoltaic performance. Science 354, 206-209 (2016).

9. Yang, M. et al. Perovskite ink with wide processing window for scalable highefficiency solar cells. Nat. Energy 2, 17038 (2017).

10. Wang, $\mathrm{L}$. et al. A Eu ${ }^{3+}-\mathrm{Eu}^{2+}$ ion redox shuttle imparts operational durability to Pb-I perovskite solar cells. Science 363, 265-270 (2019).

11. Han, Q. et al. Additive engineering for high-performance room temperature processed perovskite absorbers with micron-size grains and microsecond range carrier lifetimes. Energy Environ. Sci. 10, 2365-2371 (2017).

12. $\mathrm{Ke}, \mathrm{W}$. et al. Employing lead thiocyanate additive to reduce the hysteresis and boost the fill factor of planar perovskite solar cells. Adv. Mater. 28, 5214-5221 (2016)

13. Alsulami, Q. A. et al. Remarkably high conversion efficiency of inverted bulk heterojunction solar cells: from ultrafast laser spectroscopy and electron microscopy to device fabrication and optimization. Adv. Energy Mater. 6, 1502356-1502363 (2016).

14. Kim, H.-S. et al. Lead iodide perovskite sensitized all-solid-state submicron thin film mesoscopic solar cell with efficiency exceeding 9\%. Sci. Rep. 2, 591 (2012).

15. Chen, Y. et al. Photon management for efficient hybrid perovskite solar cells via synergetic localized grating and enhanced fluorescence effect. Nano Energy 40, 540-549 (2017).

16. Tan, H. et al. Efficient and stable solution-processed planar perovskite solar cells via contact passivation. Science 355, 722-726 (2017).

17. Ball, J. M. \& Petrozza, A. Defects in perovskite-halides and their effects in solar cells. Nat. Energy 1, 16149 (2016).

18. Huang, Y., Sun, Q. D., Xu, W., He, Y. \& Yin, W. J. Halide perovskite materials for solar cells: a theoretical review. Acta Phys. -Chim. Sin. 33, 1730-1751 (2017).

19. Liu, N. \& Yam, C. First-principles study of intrinsic defects in formamidinium lead triiodide perovskite solar cell absorbers. Phys. Chem. Chem. Phys. 20, 6800-6804 (2018)

20. Azpiroz, J. M., Mosconi, E., Bisquert, J. \& De Angelis, F. Defect migration in methylammonium lead iodide and its role in perovskite solar cell operation. Energy Environ. Sci. 8, 2118-2127 (2015).

21. Du, M. H. Density functional calculations of native defects in $\mathrm{CH}_{3} \mathrm{NH}_{3} \mathrm{PbI}_{3}$ : effects of spin-orbit coupling and self-interaction error. J. Phys. Chem. Lett. 6 1461-1466 (2015)

22. Agiorgousis, M. L., Sun, Y. Y., Zeng, H. \& Zhang, S. Strong covalencyinduced recombination centers in perovskite solar cell material $\mathrm{CH}_{3} \mathrm{NH}_{3} \mathrm{PbI}_{3}$. J. Am. Chem. Soc. 136, 14570-14575 (2014).

23. Buin, A. et al. Materials processing routes to trap-free halide perovskites. Nano. Lett. 14, 6281-6286 (2014).

24. Stewart, R. J., Grieco, C., Larsen, A. V., Doucette, G. S. \& Asbury, J. B. Molecular origins of defects in organohalide perovskites and their influence on charge carrier dynamics. J. Phys. Chem. C. 120, 12392-12402 (2016).

25. Cao, J. et al. Identifying the molecular structures of intermediates for optimizing the fabrication of high-quality perovskite films. J. Am. Chem. Soc. 138, 9919-9926 (2016).

26. Meggiolaro, D. et al. Iodine chemistry determines the defect tolerance of leadhalide perovskites. Energy Environ. Sci. 11, 702-713 (2018).

27. Liu, Z. et al. Chemical reduction of intrinsic defects in thicker heterojunction planar perovskite solar cells. Adv. Mater. 29, 1606774 (2017).

28. Zhang, W. et al. Enhanced optoelectronic quality of perovskite thin films with hypophosphorous acid for planar heterojunction solar cells. Nat. Commun. 6, 10030 (2015)

29. Yang, W. S. et al. Iodide management in formamidinium-lead-halide-based perovskite layers for efficient solar cells. Science 356, 1376-1379 (2017).

30. Niu, G. et al. Study on the stability of $\mathrm{CH}_{3} \mathrm{NH}_{3} \mathrm{PbI}_{3}$ films and the effect of post-modification by aluminum oxide in all-solid-state hybrid solar cells. J. Mater. Chem. A 2, 705-710 (2014).

31. Wang, S., Jiang, Y., Juarez-Perez, EmilioJ., Ono, LuisK. \& Qi, Y. Accelerated degradation of methylammonium lead iodide perovskites induced by exposure to iodine vapour. Nat. Energy 2, 16195 (2016).

32. Truesdale, V. W., Luther, G. W. \& Greenwood, J. E. The kinetics of iodine disproportionation: a system of parallel second-order reactions sustained by a multi-species pre-equilibrium. Phys. Chem. Chem. Phys. 5, 3428-3435 (2003)

33. Zhang, W. et al. Ultrasmooth organic-inorganic perovskite thin-film formation and crystallization for efficient planar heterojunction solar cells. Nat. Commun. 6, 6142 (2015).

34. Kubicki, D. et al. Phase segregation in Cs-, Rb- and K-doped mixed-cation $(\mathrm{MA})_{\mathrm{x}}(\mathrm{FA})_{1-\mathrm{x}} \mathrm{PbI}_{3}$ hybrid perovskites from solid-state NMR. J. Am. Chem. Soc. 139, 14173-14180 (2017).

35. Jiang, Q. et al. Planar-structure perovskite solar cells with efficiency beyond 21\%. Adv. Mater. 29, 1703852 (2017) 
36. Chen, Q. et al. Controllable self-induced passivation of hybrid lead iodide perovskites toward high performance solar cells. Nano. Lett. 14, 4158-4163 (2014).

37. Abdi-Jalebi, M. et al. Maximizing and stabilizing luminescence from halide perovskites with potassium passivation. Nature 555, 497-501 (2018).

38. $\mathrm{Bu}, \mathrm{T}$. et al. A novel quadruple-cation absorber for universal hysteresis elimination for high efficiency and stable perovskite solar cells. Energy Environ. Sci. 10, 2509-2515 (2017).

39. Wang, L. et al. Alkali metal halide salts as interface additives to fabricate hysteresis-free hybrid perovskite-based photovoltaic devices. ACS Appl. Mater. Inter. 8, 23086-23094 (2016).

40. Zheng, G. et al. Manipulation of facet orientation in hybrid perovskite polycrystalline films by cation cascade. Nat. Commun. 9, 2793 (2018).

41. Wu, X. et al. Trap states in lead iodide perovskites. J. Am. Chem. Soc. 137, 2089-2096 (2015).

42. Wright, A. D. et al. Electron-phonon coupling in hybrid lead halide perovskites. Nat. Commun. 7, 11755 (2016).

43. Mamun, A. A., Ava, T. T., Jeong, H. J., Jeong, M. S. \& Namkoong, G. A. deconvoluted PL approach to probe the charge carrier dynamics of the grain interior and grain boundary of a perovskite film for perovskite solar cell applications. Phys. Chem. Chem. Phys. 19, 9143-9148 (2017).

44. Miller, O. D., Yablonovitch, E. \& Kurtz, S. R. Strong internal and external luminescence as solar cells approach the Shockley-Queisser limit. IEEE J. Photovolt. 2, 303-311 (2012).

45. Pazos-Outón, L. M. et al. Photon recycling in lead iodide perovskite solar cells. Science 351, 1430-1433 (2016).

46. Braly, I. L. et al. Hybrid perovskite films approaching the radiative limit with over $90 \%$ photoluminescence quantum efficiency. Nat. Photon. 12, 355-361 (2018). (2018).

47. Ye, S. et al. A breakthrough efficiency of $19.9 \%$ obtained in inverted perovskite solar cells by using an efficient trap state passivator $\mathrm{Cu}$ (thiourea)I. J. Am. Chem. Soc. 139, 7504-7512 (2017).

48. Duan, H. S. et al. The identification and characterization of defect states in hybrid organic-inorganic perovskite photovoltaics. Phys. Chem. Chem. Phys. 17, 112-116 (2015).

49. Shao, Y., Xiao, Z., Bi, C., Yuan, Y. \& Huang, J. Origin and elimination of photocurrent hysteresis by fullerene passivation in $\mathrm{CH}_{3} \mathrm{NH}_{3} \mathrm{PbI}_{3}$ planar heterojunction solar cells. Nat. Commun. 5, 5784 (2014).

50. De Wolf, S. et al. Organometallic halide perovskites: sharp optical absorption edge and its relation to photovoltaic performance. J. Phys. Chem. Lett. 5, 1035-1039 (2014).

51. Dong, Q. F. et al. Electron-hole diffusion lengths $>175 \mu \mathrm{m}$ in solution-grown CH3NH3PbI3 single crystals. Science 347, 967-970 (2015).

52. Xing, G. et al. Low-temperature solution-processed wavelength-tunable perovskites for lasing. Nat. Mater. 13, 476-480 (2014).

53. Green, M. A. et al. Solar cell efficiency tables (Version 45). Prog. Photovolt. Res. Appl. 26, 3-12 (2018).

54. Zhou, H. P. et al. Interface engineering of highly efficient perovskite solar cells. Science 345, 542-546 (2014).

55. Bi, D. et al. Efficient luminescent solar cells based on tailored mixed-cation perovskites. Sci. Adv. 2, 1501170 (2016).

56. Clark, S. J. et al. First principles methods using CASTEP. Z. Krist. 220, 567-570 (2005).

57. Perdew, J. P. \& Wang, Y. Accurate and simple analytic representation of the electron- gas correlation energy. Phys. Rev. B 45, 13244-13249 (1992).

58. Probert, M. I. J. \& Payne, M. C. Improving the convergence of defect calculations in supercells: an ab initio study of the neutral silicon vacancy. Phys. Rev. B 67, 075204 (2003).
59. de Mello, J. C., Wittmann, H. F. \& Friend, R. H. An improved experimental determination of external photoluminescence quantum efficiency. Adv. Mater. 9, 230-232 (1997)

60. Wang, N. et al. Perovskite light-emitting diodes based on solutionprocessed self-organized multiple quantum wells. Nat. Photon. 10, 699-704 (2016).

\section{Acknowledgements}

This work is supported by the National Natural Science Foundation of China (51722201) (51672008) (91733301), Beijing Natural Science Foundation (4182026), National Key Research and Development Program of China Grant no. 2017YFA0206701, and Young Talent Thousand Program.

\section{Author contributions}

H.P.Z., Y.H.C. and Q.C. conceived and coordinated the project. Y.H.C. and H.Y.J. conducted UV-vis spectra and SEM characterizations. Y.H.C., N.X.L., L.G.W., L.L. and Z.Q.X. carried out the solar cell fabrication, optimization, and certification in Newport PV Lab. Y.H.C. and L.L. measured the PL spectra. Y.H.C. and P.F.L. contributed to the thermal admittance spectra and analysis. W.Z. and J.P.W. contributed to the PLQE measurement. Y.H.C., C.Z. and H.C.Z. conducted a transient photovoltage decay curve, a photocurrent decay curve, and light intensity-dependent $V_{\mathrm{OC}}$ measurement. M.Z.S., B.L.H. and D.D.L. helped with theoretical simulation and in-depth discussion. S.Z., G.C.X., and X.F.L. contributed to femtosecond optical measurement and calculation. All authors assisted in the interpretation of results. Y.H.C. wrote the paper, and H.P.Z. and Q.C. helped with editing.

\section{Additional information}

Supplementary Information accompanies this paper at https://doi.org/10.1038/s41467019-09093-1.

Competing interests: The authors declare no competing interests.

Reprints and permission information is available online at http://npg.nature.com/ reprintsandpermissions/

Journal peer review information: Nature Communications thanks E.K. Kim and the other anonymous reviewer(s) for their contribution to the peer review of this work.

Publisher's note: Springer Nature remains neutral with regard to jurisdictional claims in published maps and institutional affiliations.

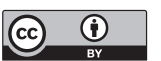

Open Access This article is licensed under a Creative Commons Attribution 4.0 International License, which permits use, sharing, adaptation, distribution and reproduction in any medium or format, as long as you give appropriate credit to the original author(s) and the source, provide a link to the Creative Commons license, and indicate if changes were made. The images or other third party material in this article are included in the article's Creative Commons license, unless indicated otherwise in a credit line to the material. If material is not included in the article's Creative Commons license and your intended use is not permitted by statutory regulation or exceeds the permitted use, you will need to obtain permission directly from the copyright holder. To view a copy of this license, visit http://creativecommons.org/ licenses/by/4.0/.

(C) The Author(s) 2019 